\title{
Von der Idee zum Unternehmen
}

\author{
Selbstständigkeit in der Sozialen Arbeit \\ erfordert einen professionellen Businessplan
}

\author{
Thomas Müller
}

\begin{abstract}
A good professional concept is no guarantee for successful self-employment in the area of social work. What is needed is a map for finding the way to the own enterprise: a business plan.
\end{abstract}

Ein gutes fachliches Konzept ist keine $G a$ rantie für eine erfolgreiche Selbstständigkeit in der Sozialen Arbeit. Gebraucht wird eine Landkarte für den Weg zum eigenen Unternehmen: der Businessplan.

Die Unzufriedenheit vieler professioneller Fachkräfte mit ihrer Arbeitssituation in Sozialverwaltungen und bei freien Trägern wächst. Einstellungsträger als Arbeitgeber verlieren an Bedeutung, weil von Seiten der Leistungsträger eine Kostenreduktion intendiert wird. In dieser Situation gewinnt Selbstständigkeit für viele Fachkräfte der Sozialen Arbeit an Attraktivität; als Vorteile werden das eigenständige und flexible Arbeiten und die Freiheit in der Arbeitsgestaltung gesehen. Selbstständigkeit erscheint als Gegensatz einer »abhängigen Tätigkeit « (vgl. $\$ 7$ Abs. 1 SGB IV und $\mathbb{S} 84$ Abs. 1 HGB). Allerdings sollte neben den Chancen, die eine Selbstständigkeit bietet, auch die Risiken betrachtet werden. Diese liegen insbesondere im finanziellen Bereich, da viele Sozialarbeiter nicht über die nachfolgend skizzierten betriebswirtschaftlichen Kenntnisse verfügen, die ein solcher Schritt erfordert.

Neben Fragen zur eigenen Motivation, die ehrlich und selbstkritisch beantwortet werden sollten, stellt vor allem die finanzielle Situation ein wichtiger Eckpfeiler zu einer gesicherten Existenz dar und sollte daher zwingend kalkuliert werden. Insbesondere das Startkapital und eine Durststrecke zu Beginn sind zu bedenken. Gerade bei Neugründungen im Sozialbereich ist der Kapitalbedarf höchst unterschiedlich. Die Spanne reicht dabei von wenigen hundert bis tausend Euro im Tagesmütterbereich bis $\mathrm{zu}$ mehreren zehntausend Euro, wenn beispielhaft ein Kindergarten in angemieteten Räumen betrieben werden soll.

Das erforderliche Kapital kann, wenn nicht schon vorhanden, zum einen über die Hausbank, die Bank für Sozialwirtschaft, die Kreditanstalt für Wiederauf- bau o. Ä. beschafft werden, zum anderen über Förderprogramme beispielsweise der Europäischen Union, des Bundes oder der Länder. Förderungen können projekt- oder institutionsbezogen gewährt werden. Auch Instrumente wie Fundraising, Sponsoring oder Lotteriemittel (z. B. »Aktion Mensch «) sollte man beachten (vgl. Nicolini 2006, 131 ff.).

Wichtige Vorbereitung für die Kapitalsuche ist ein Businessplan. Dieser fasst das Vorhaben schriftlich zusammen. Potenzielle Kapitalgeber können so die Idee und das Konzept detailliert beurteilen. Der Businessplan, der nicht länger als 25 bis 30 Seiten sein sollte, ist meistens wie folgt gegliedert (nach Peters/Bierwirth 2007, 20 f.):

- Die Geschäftsidee: Der Einstieg soll das Interesse des Kapitalgebers wecken. Inhalte sind die Art »der Dienstleistung und der Kundennutzen, die fachliche Qualifikation des Managements, der Investitionsbedarf mit möglicher Rendite sowie die Chancen und Risiken « (Peters/Bierwirth 2007, 20).

- Das Unternehmen: Bestandteile dieses Punktes sind die rechtliche Struktur, Erfahrungen und Qualifikationen der Gründer, die Standortwahl, die etwaige Vorgeschichte des Gründungsunternehmens und das voraussichtliche Wachstum.

- Das Leistungsangebot: Hier werden insbesondere die Vorteile des Konzeptes gegenüber bereits am Markt vorhandenen Angeboten aufgeführt (z. B. Kostenvorteile für die Kunden, Zusatznutzen, fachlich-inhaltliche Weiterentwicklung etc.).

- Der Markt und der Wettbewerb: Gefragt sind Aussagen über Konkurrenten, Kunden und die Rahmenbedingungen des Marktes. Es ist eine Marktanalyse durchzuführen, die eine Wettbewerbsanalyse beinhalten sollte, in der ein Gesamtüberblick über die Lage im angestrebten Segment und eine Analyse der Hauptkonkurrenten er- 
stellt wird. Mögliche Kooperationspartner, die entweder bereits am Markt agieren oder sich mit dem Gedanken tragen, dies zu tun, werden analysiert. Dieses Kapitel muss ein realistisches Bild der aktuellen Situation des Geschäftsfeldes zeichnen. In Kombination mit Statistiken und persönlichen Ein-
Zeit, die Ergebnisse in eine sogenannte Wirtschaftsplanung umzusetzen. Intention hierbei ist es zu zeigen, dass die Ideen und Konzepte bezahlbar und rentabel sind. Inhalte sind beispielsweise ein Liquiditätsplan, eine Umsatz- und Erlösprognose, eine Personal- und Investitionsplan etc.

\section{»Bei Existenzgründungen im Sozialbereich ist der Kapitalbedarf böchst unterschiedlich«}

schätzungen können Prognosen künftiger Entwicklungen aufgestellt werden. Die gewonnenen Erkenntnisse bilden die Basis für die Planung strategischer Maßnahmenbündel. Handlungsleitende Fragen für die Marktanalyse sind beispielsweise: Wie groß ist der potenzielle Bedarf im fokussierten Einzugsgebiet? Gibt es bereits Anbieter dieser Dienstleistung am Markt, wenn ja, wie viele? Wie sind diese Anbieter einzuschätzen (starker Wettbewerber etc.)? Existieren Angebotslücken? Gibt es vor- oder nachgelagerte Institutionen, die sich als Kooperationspartner anbieten?

- Das Marketing und der Vertrieb: Das im vorhergehenden Punkt prognostizierte Marktpotenzial muss erschlossen werden. Dazu wird ein Marketing- und Vertriebskonzept benötigt, in dem Ziele, Zielgruppen, Vorgehensweisen und Maßnahmen zur Absatzförderung (z. B. Inserate, Tage der offenen Tür etc.) aufgeführt werden.

- Die Organisation: Wichtiges Element eines zukunftsträchtigen Konzeptes ist die Darstellung der Aufbau- und Ablauforganisation. Anfangs mag dies keine große Rolle spielen, aber mit steigendem Personal- und Kundenbestand steigt auch die Wichtigkeit der Betriebsorganisation. Denn Fragen nach der Aufgabenteilung, der Informationsweitergabe und die Festlegung von Verantwortungsbereichen sind in Organisationen essenziell. Ferner sollten die fachlich-inhaltlichen, wie auch die betriebswirtschaftlichen Kompetenzen des Gründers illustriert werden.

- Die Fünfjahresplanung: Nach den bisherigen Überlegungen ist es nun an der
- Der Finanzbedarf: Beschrieben werden müssen die unterschiedlichen Finanzierungsquellen (z. B. Kredite, Förderungen, Zuschüsse etc.). Hierbei werden die Finanzierungsarten (Fremd- oder Eigenfinanzierung) und die jeweiligen Laufzeiten aufgeschlüsselt (vgl. Peters/Bierwirth 2007, 20 f.).

Eine zündende Idee, ein gutes fachliches Konzept allein sind kein Garant für eine erfolgreiche Selbstständigkeit. Erst im Zusammenspiel mit einer soliden finanzwirtschaftlichen Planung kann aus einer »Vision im Geiste « eine »Existenz in der Realität « werden. Das zentrale Instrument dafür ist ein aussagekräftiger Businessplan. Eine externe Existenzgründungsberatung kann zum einen bei der Erstellung eines solchen Businessplanes helfen und zum anderen einschlägige Förderprogramme ausloten.

\section{Warnung}

Noch etwas ist zu bedenken: Für die Profession wird es künftig darum gehen, einen Mindestlohn für professionelle Soziale Arbeit durchzusetzen. Es kann nicht im Interesse der in der Sozialbranche Tätigen sein, dass letztlich ein fest angestellter Mitarbeiter mit seinem (Tarif-) Lohn gegen seinen selbstständigen Kollegen ausgespielt wird.

Hier gilt es, Zusammenschlüsse und Gremien zu bilden, die sich organisiert und vernetzt für die Belange einer fachgerechten professionellen Arbeit einsetzen und Standards bilden. Denn auch Beratung in rechtlichen Fragen, beispielsweise zu Musterverträgen, Versicherungs- und Steuerfragen ist im Bereich der Selbst- ständigkeit von hoher Wichtigkeit. Gruppen wie die Bundesfachgruppe Selbstständige des Deutschen Berufsverbandes für Soziale Arbeit e. V. (vgl. Seite 218), die sich solcher Themen annehmen, sind sehr zu begrüßen.

\section{Literatur}

Handelsgesetzbuch (HGB) in der Fassung vom 10. Januar 2007.

Maier, Konrad/Spatscheck, Christian 2006: Wider das ritualisierte Klagelied über die schlechten Arbeitsmarktchancen für SozialarbeiterInnen. In: Sozial Extra 11/2006, 26-33.

Nicolini, Hans J. 2006: Finanzierung für Sozialberufe. Grundlagen - Beispiele - Übungen.

Peters, André/Bierwirth, Maike 2007: Erfolgreich finanziert?! Klassische und neue Finanzierungsformen für die Sozialwirtschaft. Contec $\mathrm{GmbH}$ (Hg.).

Sozialgesetzbuch Viertes Buch (SGB IV) in der Fassung vom 23. Juli 2004.

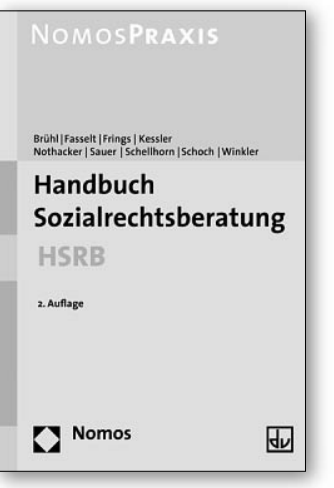

\section{Handbuch Sozialrechts- beratung - HSRB}

Von RA Prof. Dr. Albrecht Brühl, Prof. Dr. Ursula Fasselt, Prof. Dr. Dorothee Frings, Prof. Dr. Rainer Kessler, Prof. Dr. Gerhard Nothacker, Prof. Dr. Jürgen Sauer, Prof. Dr. Helmut Schellhorn, RegDir a.D. Dietrich Schoch und Prof. Dr. Jürgen Winkler 2. Auflage 2007, 666 S., brosch., $44,-€$, ISBN 978-3-8329-2382-2

\section{Nomos}

